\title{
New proposal for skin grafts on tendon-exposed wounds
}

\author{
Jung Hwan Um, Dong In Jo, Soon Heum Kim \\ Department of Plastic and Reconstructive Surgery, Konkuk University Chungju Hospital, Konkuk University School of Medicine, Chungju, \\ Korea
}

\begin{abstract}
Performing a skin graft is not feasible for tendon-exposed defect reconstruction because tendons are fibrous connective tissues with relatively poor blood supply. This study proposes a method to effectively perform skin graft surgery in tendon-exposed wounds. A 48-year-old male patient with diabetes mellitus had a very large left dorsal foot defect $(8 \times 8 \mathrm{~cm})$. The wound bed had healthy granular tissue, with tendon exposure. The tendons were turned over so that the posterior side would behave as the anterior side. The edge of the paratenon was then fixed together to the surrounding granulation tissue or dense remnant fascia using absorbable sutures, and the close granulation tissue was approximated and buried. A splitthickness skin graft was performed after 1 week. The graft site was stably taken on postoperative day 3. A small disruption was then observed at the graft site within 1 week postoperatively, but conservative treatment was continued for 1 month, after which the defect site was completely restored. This technique can increase the success rate of skin grafts for defects with tendon exposure.
\end{abstract}

Keywords Skin graft / Granulation / Tendon / Diabetic foot / Turn over

\author{
Correspondence: Soon Heum Kim \\ Department of Plastic and \\ Reconstructive Surgery, Konkuk \\ University Chungju Hospital, Konkuk \\ University School of Medicine, \\ 82 Gugwon-daero, Chungju 27376, \\ Korea \\ Tel: $+82-43-840-8860$ \\ Fax: +82-43-840-9862 \\ E-mail: plastics7@kku.ac.kr
}

The authors thank Jiyoung Kim (zlawldud226@gmail.com) for clear anatomical illustrations.

Received: February 7, 2021 • Revised: August 21, $2021 \bullet$ Accepted: September 29, 2021

pISSN: 2234-6163 • elSSN: 2234-6171 • https://doi.org/10.5999/aps.2021.00297 • Arch Plast Surg 2022;49:86-90

\section{INTRODUCTION}

Skin defects are a common type of trauma treated by plastic and reconstructive surgeons. Epithelialization can be induced through secondary intention or primary closure in cases of small skin defects. However, surgical reconstruction is essential for large defects or if tendons or bones are exposed. Several surgical options are available. Skin graft or flap surgery is commonly used in such circumstances. Skin grafts are beneficial in terms of the ability to harvest and graft skin through a simple procedure, and they can be used to cover wide defects. However, a drawback is that their prognosis is dependent to a great extent on the condition of the wound bed because skin graft procedures do not involve concomitant transplantation of blood vessels [1]. In contrast, flap surgery can safely cover skin defects despite poor wound bed conditions because the flap receives its own blood supply through the blood vessels included in the flap. However, flap surgery is generally a more difficult procedure than skin graft surgery because it involves microsurgery. In addition, new defects can occur at the flap donor site, and more serious complications can occur if flap surgery fails [2].

The dorsal surface of the hands and feet has thin, soft tissue characterized by an anatomical structure in which the extended tendons are directly under the soft tissue. Thus, tendons are more commonly exposed on the dorsal surface than when skin defects occur on the palmar/plantar surface. The failure rate is 
reported to be very high when skin grafts are performed on tendon-exposed wound beds because tendons are fibrous connective tissues with a relatively poor blood supply [3-5]. However, flap surgery also shows a poor prognosis in patients with diabetes who have poor peripheral blood vessels [6]. Thus, this study presents a surgical technique that can increase the success rate of skin grafts on tendon-exposed defects.

\section{IDEA}

A burn wound on the left dorsal foot of a 48-year-old male patient with diabetes mellitus (DM) caused by hot water persisted for 1 month without healing, and an $8 \times 8 \mathrm{~cm}$ full-thickness skin defect was observed with exposed tendons. This patient was receiving outpatient follow-up for DM neuropathy. Before the injury, the sensory and motor functions of the foot, particularly the toes, were impaired. Normal motion of the ankle was noted at the patient's first visit to the department of plastic and reconstructive surgery, but all toes could not be extended, being flexed. The extensor hallucis longus, the tibialis anterior tendon, and the extensor digitorum longus were the exposed tendons. At first, purulent discharge and severe erythema were observed, and antibiotic treatment, debridement, and negative pressure wound therapy (NPWT) were regularly performed. NPWT was performed continuously at a pressure of $125 \mathrm{mmHg}$ and was changed every 3 days. Consequently, a healthy wound bed

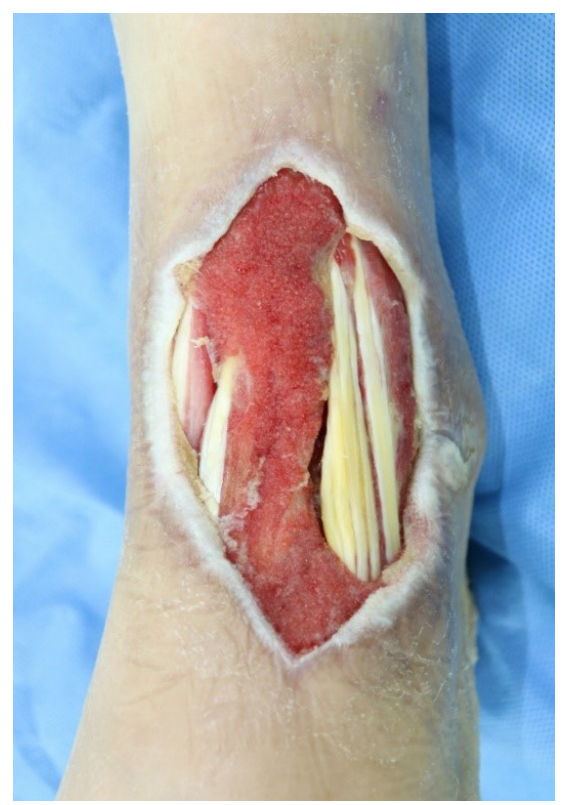

Fig. 1. Preoperative photograph of dorsal view. An $8 \times 8 \mathrm{~cm}$ full-layer skin defect was observed with exposed tendons in a 48-year-old male patient with diabetes mellitus. From the left, the extensor hallucis longus, the tibialis anterior tendon, and the extensor digitorum longus tendons were exposed. covered with granulation tissue was observed after 1 month (Fig. 1). However, the granulation tissue could not grow in the tendon-exposed areas. The tendon sheaths on the dorsal side of the tendons were all lost, and only the fibers of the tendons were exposed. However, when the tendons were turned over, the posterior surfaces of the tendons were observed to be positioned outward and covered with healthy granulation tissue, unlike the exposed dorsal surfaces. The posterior surfaces of the exposed tendons were attached to the adjacent granulation tissue and could not be easily elevated. The tendons were turned over so that the posterior surface could behave as the anterior side. The edge of the paratenon was then fixed together to the surrounding granulation tissue or dense remnant fascia using 5-0 Vicryl sutures, and the close granulation tissue was approximated and buried (Fig. 2). During this procedure, although minimal bleeding was observed along the margin of the turnedover tissue, it was not actively controlled for vascularity. Only granulation tissue was observed on the wound bed, without tendon exposure. After performing simple dressing changes daily with antibiotic ointment for 1 week, a split-thickness skin graft was performed. The graft was harvested from the left thigh and laid on the granulation tissue (Fig. 3). A tie-over dressing was performed and a short leg splint was applied for 2 weeks.

The skin graft site was observed to be stable 3 days after the skin graft operation. This was followed by simple dressing changes using foam material daily. A small disruption $(1 \times 2 \mathrm{~cm})$

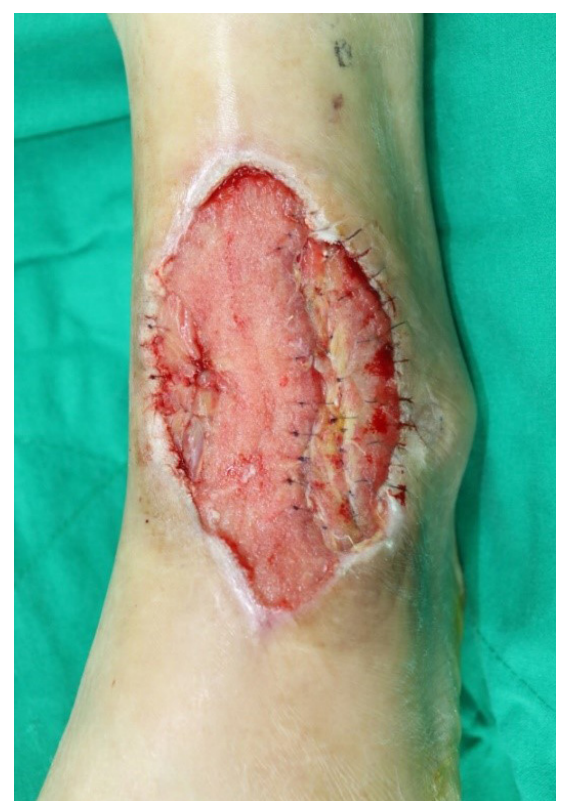

Fig. 2. Intraoperative photograph of dorsal view. The tendons were turned over so that the posterior surface could behave as the anterior side and fixed together to the surrounding granulation tissue or dense remnant fascia using 5-0 Vicryl sutures. 
was observed in the graft site where the tendon had been exposed on postoperative day 7 . Conservative treatment was maintained for 1 month, after which the defect site was completely restored (Fig. 4). Compared with the preoperative movements, similar motion of the ankle was observed, but no change was observed in the extension of the toes. After complete wound healing, a silicone sheet was applied to prevent the occurrence of hypertrophic scars, and rehabilitation exercises

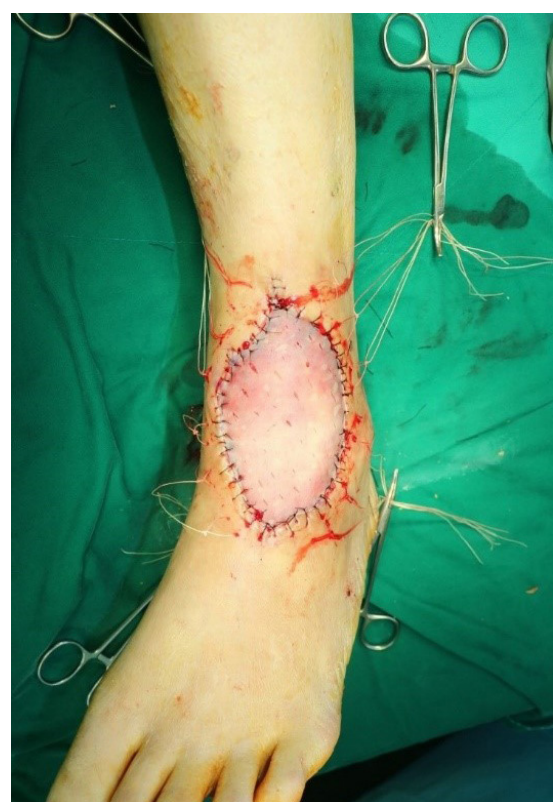

Fig. 3. Intraoperative photograph of dorsal view. A split-thickness skin graft was performed 1 week after the tendon turnover technique. were performed to improve the motor function of the foot upon consultation with the department of rehabilitation medicine. The patient remains in a stable condition to date.

\section{DISCUSSION}

Skin defects are common injuries treated by plastic and reconstructive surgeons, and according to the reconstructive ladderthat is, the concept that reconstruction should initially be considered with a relatively simple surgical procedure-skin graft surgery can be considered before flap surgery for defect coverage. Because flap surgery transfers tissue components together with blood vessels, the recipient's vascular status must be considered. Thus, flap surgery in patients with comorbidities who have poor systemic vascular status may be dangerous [6]. In addition, the donor-site morbidity in flap surgery should be considered, because if this procedure fails, more serious complications may occur. In contrast, an advantage of skin grafts is a relatively simple operation that involves free tissue transfer of only skin to the wound bed without blood vessel transfer. However, for this reason, a skin graft cannot be considered if the condition of the wound bed is poor because the nutrients and oxygen are delivered via diffusion, such as plasma imbibition, until revascularization occurs [7-9]. In the case described herein, if a skin graft had been laid over the tendons, which had a poor blood supply, this procedure would have been very likely to fail.

The vascular supply of tendons has three main components. The first is the musculotendinous junction, the second is the os-
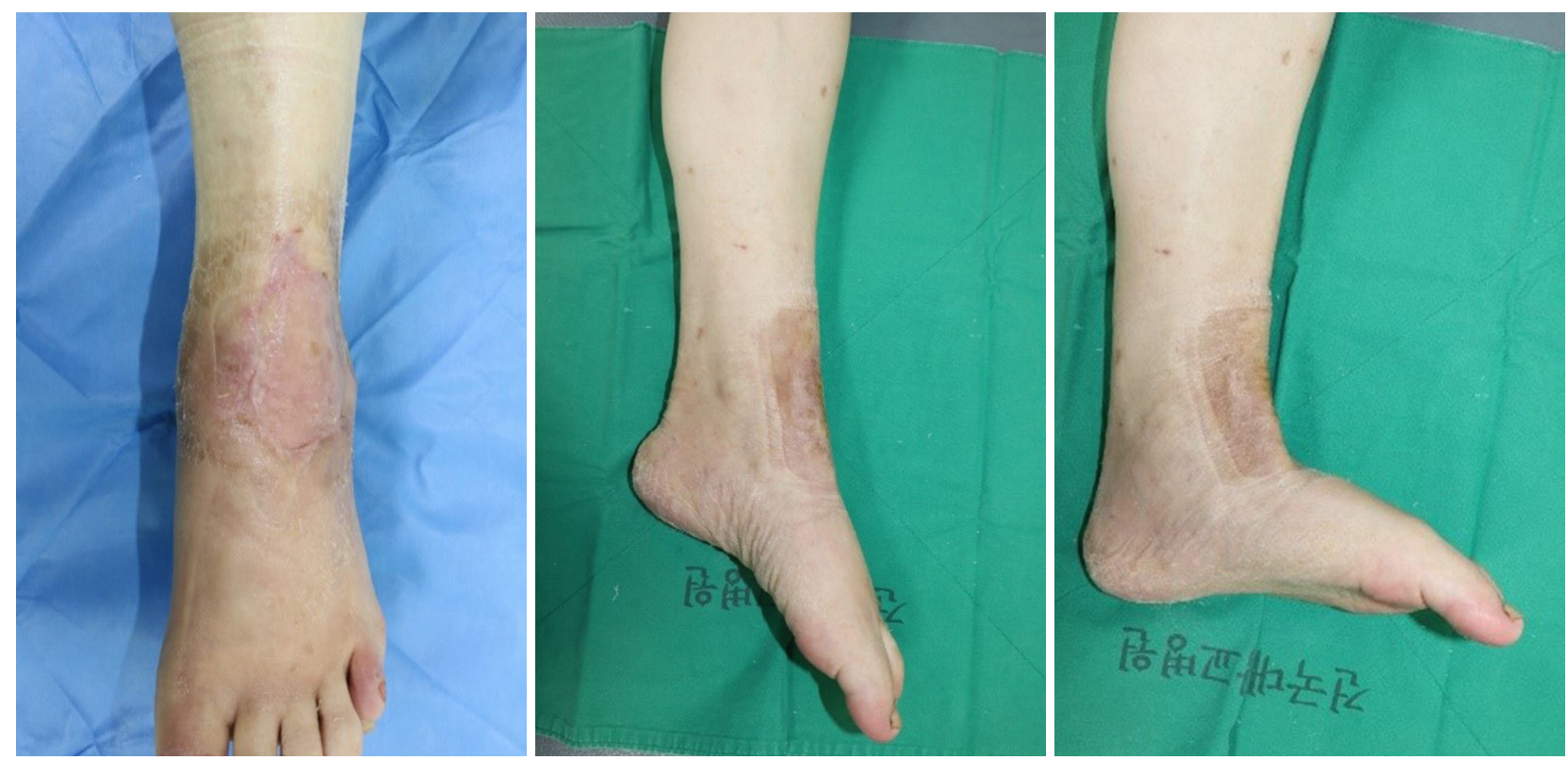

Fig. 4. The skin defect was completely reconstructed 1 month after surgery. The motion of the ankle was similar to its preoperative range. (A) Dorsal view. (B) Plantar flexion was possible. (C) Dorsiflexion was possible. 
teotendinous junction, and, finally, the vascular supply is directly received from the vessels of various surrounding connective tissues such as the paratenon, mesotenon, and vincula $[3,5,10$, 11]. However, in most parts, tendons are poorly vascularized tissues that rely more on synovial fluid diffusion than vascular perfusion for the supply of nutrients and oxygen [12]. Diffusion of synovial fluid mostly occurs from the tendon sheath and surrounding connective tissues with abundant blood vessels, unlike the tendons.

A limitation of this study is that it presents a single case. Nevertheless, this method can be applied to sufficiently diverse cases if one side of the tendon is in contact with the viable granulation tissue. Although defects with tendon exposure have various causes, such as trauma, burns, and secondary lesions induced by comorbidities, the same result is theoretically expected if the posterior side of the exposed tendon is in contact with the granulation tissue. This expectation is rooted in an in-depth understanding of the anatomy of the vascular system of the tendons and surrounding connective tissues. In this case, only dry fibrous tissue without granulation tissue was observed on the anterior surface with tendon exposure. However, the posterior surface of the tendon, which remained in constant contact with the granulation tissue, preserved the tendon sheath with abundant vascularity, presenting an appropriate environment for the growth of granulation tissue. The key point in this study is to

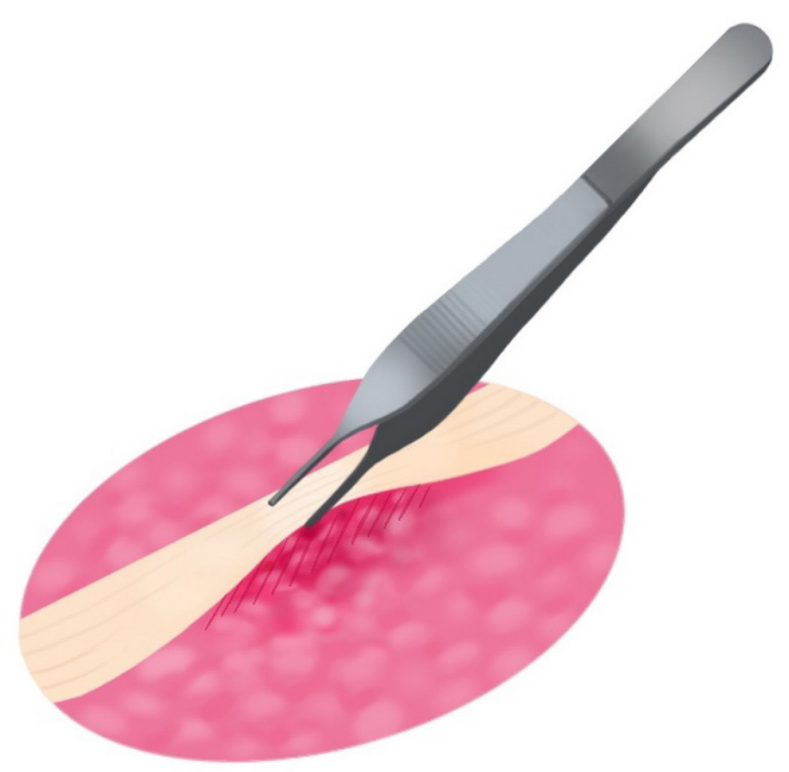

Fig. 5. Illustration of the posterior surface of an exposed tendon. Connective tissue with abundant vascularity was observed when the tendons were turned over and the posterior surfaces of the tendons were observed facing outward. turn over the tendon to create a favorable environment for the graft to be taken.

The study started with a simple observation: connective tissue with abundant vascularity was noted when the tendons were turned over and the posterior surfaces of the tendons were observed facing outward (Fig. 5). This connective tissue is assumed to have been the remnant tissue of the paratenon and synovial tissue surrounding the tendons. The anterior and posterior sides of the tendons were turned over, exposing the side with abundant blood supply outward because the tendon-exposed defect had poor blood supply (Fig. 6). The skin graft was stably taken, and this method can be applied to skin defects in many areas of the hands and feet where tendons are exposed. Moreover, recovery of the patient's ankle joint in terms of its range of motion was observed after the skin graft was taken. However, because most tendon-exposed defects are near a joint, a skin graft becomes unstable during movement, and the probability of hypertrophic scars in the graft-taken area may increase. Because the range of motion may be limited due to hypertrophic scars or contracture at the graft site, it would be essential to conduct early scar management and rehabilitation to prevent these issues in advance.

This method is a novel proposal for simple wound coverage in patients with comorbidities, in whom it is difficult to consider flap surgery, or for skin defects with exposed tendons.

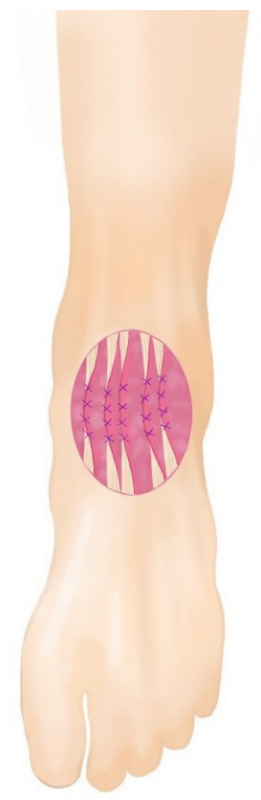

Fig. 6. Illustration of the technique of turning over the tendons. The tendons were turned over so that the posterior surface could behave as the anterior side. The edge of the paratenon was then fixed together to the surrounding granulation tissue or dense remnant fascia using absorbable sutures, and the close granulation tissue was approximated and buried. 


\section{NOTES}

\section{Conflict of interest}

No potential conflict of interest relevant to this article was reported.

\section{Ethical approval}

The study was approved by the Institutional Review Board of Konkuk University Chungju Hospital (IRB No. KUCH 202010-027) and performed in accordance with the principles of the Declaration of Helsinki.

\section{Patient consent}

The patient provided written informed consent for the publication and the use of his images.

\section{Author contribution}

Writing - original draft: JH Um. Writing - review \& editing: JH Um, DI Jo, SH Kim. Conceptualization: DI Jo, SH Kim. All authors read and approved the final manuscript.

\section{ORCID}

Jung Hwan Um

https://orcid.org/0000-0002-9129-5582

Dong In Jo https://orcid.org/0000-0002-3075-4482

Soon Heum Kim

\section{REFERENCES}

1. Aerden D, Bosmans I, Vanmierlo B, et al. Skin grafting the contaminated wound bed: reassessing the role of the preop-

erative swab. J Wound Care 2013;22:85-9.

2. Park JW. Reconstruction of a traumatic soft tissue defect. J Korean Fract Soc 2015;28:256-65.

3. Takasugi H, Akahori O, Nishihara K, et al. Three-dimensional architecture of blood vessels of tendons demonstrated by corrosion casts. Hand 1978;10:9-15.

4. Schmidt-Rohlfing B, Graf J, Schneider U, et al. The blood supply of the Achilles tendon. Int Orthop 1992;16:29-31.

5. Ahmed IM, Lagopoulos M, McConnell P, et al. Blood supply of the Achilles tendon. J Orthop Res 1998;16:591-6.

6. Lese I, Biedermann R, Constantinescu M, et al. Predicting risk factors that lead to free flap failure and vascular compromise: a single unit experience with 565 free tissue transfers. J Plast Reconstr Aesthet Surg 2021;74:512-22.

7. Scherer S, Pietramaggiori G, Orgill DP. Skin graft. In: Neligan PC, editor. Plastic surgery. 3rd ed. New York: Elsevier; 2012. p. 323-4.

8. Blume PA. Skin grafts. In: Dockery GD, Crawford ME. Low extremity soft tissue \& cutaneous plastic surgery. Edinburgh: Elsevier; 2012. p. 207.

9. Kim JB, Han SK, Kim WK. Deepithelialized split thickness skin graft. J Korean Wound Care Soc 2006:2:34-40.

10. Chansky HA, Iannotti JP. The vascularity of the rotator cuff. Clin Sports Med 1991;10:807-22.

11. Brockis JG. The blood supply of the flexor and extensor tendons of the fingers in man. J Bone Joint Surg Br 1953;35B:131-8.

12. Gelberman RH. Flexor tendon physiology: tendon nutrition and cellular activity in injury and repair. Instr Course Lect 1985;34:351-60. 